\title{
THE CIRCUMSTELLAR ENVIRONMENT OF CHROMOSPHERICALLY ACTIVE T TAURI STARS
}

\author{
U. Finkenzeller and G. Basri \\ Astronomy Department \\ University of California \\ Berkeley, CA 94720
}

\begin{abstract}
We discuss new spectroscopic material on $7 \mathrm{~T}$ Tauri stars of low to intermediate activity level which have envelopes of low optical thickness and small circumstellar/interstellar extinction. We show that difference plots between the target star and appropriate standards are a powerful tool to probe the stellar envelope structure. In our sample we find 1 object with a P Cyg type, 3 with inverse $P$ Cyg type, and 3 with symmetrical Balmer line profiles. We conclude that the physical processes in these $T$ Tauri stars do not differ qualitatively from the ones found in extremely active ones. In particular, the inverse $\mathbf{P}$ Cyg type profiles are not restricted to stars with very opaque envelopes and are possibly a much more common attribute of young stellar objects.
\end{abstract}

\section{DATA BASE}

The work discussed here is part of a comprehensive study of $\mathrm{T}$ Tauri stars with low to intermediate activity levels which were selected from the low resolution catalogue of Appenzeller et al. (1983). Our observational material is innovative since it is at high resolution ( $R=12000)$, is flux calibrated, has a wide wavelength span (3900 to $8700 \AA)$, and was obtained quasi-simultaneously. The program intentionally focuses on objects showing low to intermediate activity as referenced by weak or moderate emission line strength, i.e. the least complex manifestation of the $\mathrm{T}$ Tauri phenomenon. For more details, see Finkenzeller and Basri (1985).

\section{DIFFERENCE PLOTS AS A DIAGNOSTIC TOOL}

The arithmetic difference between a target star and an appropriate photospheric spectral standard turned out to be a powerful tool to analyze high $\mathrm{S} / \mathrm{N}$ spectrograms. For this purpose, the spectrogram of the standard has to be shifted to the rest frame of the $T$ Tauri star and convolved with a rotational function to match the $v * \sin i$ of the target star.
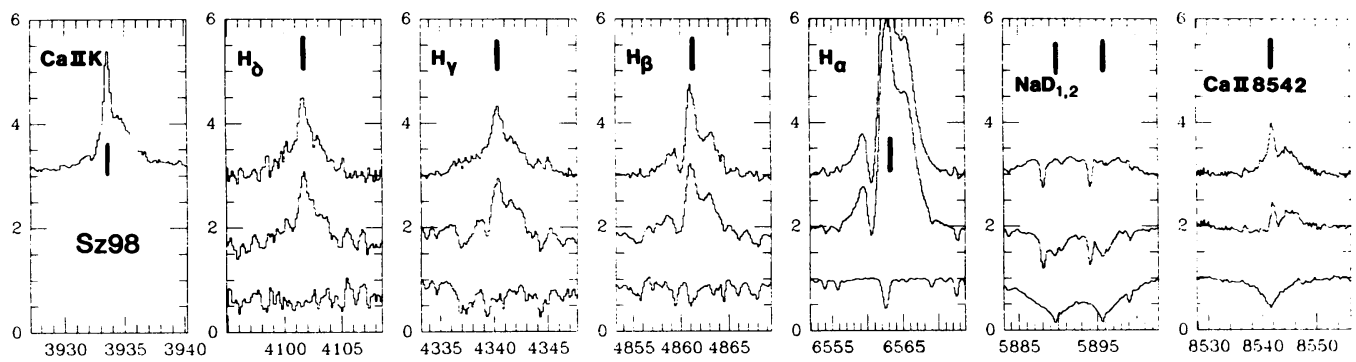

Fig. 1 For the T Tauri star Sz65 all major emission lines are given as diffference and original data (top and middle tracing). The lower tracing is the processed standard star. A vertical tick indicates the systemic velocity, and each frame is $\pm 500 \mathrm{~km} / \mathrm{s}$ wide. 

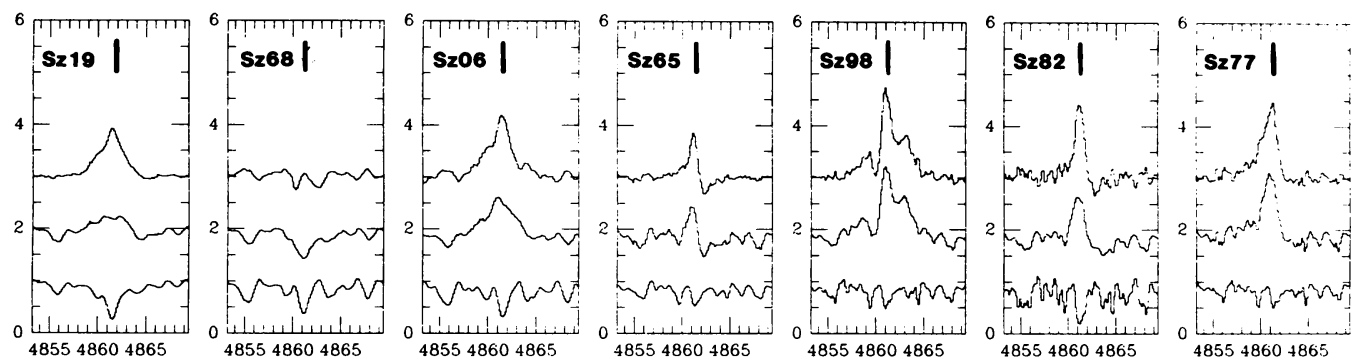

Fig. $2 H_{\beta}$ for all the T Tauri stars, same representation as in Fig. 1. From left to right, the $H_{\beta}$ surface flux $\left(\mathrm{ergs} / \mathrm{cm}^{2} \mathrm{~s}\right)$ and $A_{v}$ (mags) are 4.2e7, 2.5; n.a., 1.7; 2.6e7, 1.9; n.a., $0.9 ; 1.3 \mathrm{e} 7,0.8 ; 7.1 \mathrm{e} 6,0.9$.

Applied to the lines of $H_{\alpha}, H_{\beta}, H_{\gamma}$, and $H_{\delta}$ one can show that all line profiles closely resemble each other (Fig. 1), indicating formation in a region with common physical properties. CaII H, CaII K, and the IR triplet lines of Calcium share this property as well.

The appearance of upper photospheric and chromospheric lines when using this differential comparison method is already described by Finkenzeller and Basri (1985) and will not be discussed here.

\section{LINE PROFILE TYPES}

The selected $\mathrm{T}$ Tauri stars represent the least active species of their class, but display in the same variety of line profiles as their violently active counterparts. In particular, we were surprised to find 3 objects (Sz65, Sz82, and Sz77) whose line profiles have emission wings more steep on the red than on the blue side. A weak redshifted absorption component, vaguely present in the original data, is more distinct in the difference representation (Fig. 2) and reminiscent of the so-called YY Ori profiles (interpreted by some authors as indicative of mass infall). Sz98 shows a Beals type III P Cyg profile, whereas Sz06, Sz19, and $\mathrm{Sz68}$ have symmetrical line profiles.

Until now, the occurence of inverse $\mathrm{P}$ Cyg profiles with $\mathrm{T}$ Tauri stars was known to occur only with the most active (or extreme) $\mathrm{T}$ Tauri stars, generally thought to be the youngest in their class. The 3 low activity objects found here are only marginally reddened $\left(A_{v}=0.8-0.9 \mathrm{mag}\right)$ yet show this phenomenon. The formation of inverse $P$ Cyg profiles might not directly be related to the presence of infalling material with high column densities. The combination of radiation transport effects with non-spherical geometries (see, e.g. Ulrich and Knapp 1985 or Bertout 1979) may be a more acceptable scenario.

\section{REFERENCES:}

Appenzeller, I., Jankovics, I., Krautter, J. 1983, Astron.Astrophys.Suppl., 53, 191

Bertout, C. 1979, Astron.Astrophys., 80, 139

Finkenzeller, U., Basri, G. 1985, The ESO Messenger, 42, 20

Ulrich, R., Knapp, G. 1985, preprint 